\title{
Hepatocytes with a phenotype of substantial CYP3A4 induction generated from drug-associated fulminant hepatitis-derived induced pluripotent stem cells
}

Kenta Ite $^{1,2}$, Masashi Toyoda ${ }^{1,3}$, Saeko Yoshioka ${ }^{1}$, Takaaki Yukitake ${ }^{1,4}$, Mayu YamazakiInoue $^{1}$, Kuniko Tatsumi ${ }^{1}$, Hidenori Akutsu ${ }^{1}$, Hiroshi Nishina ${ }^{5}$, Tohru Kimura ${ }^{6}$, Naoko

Ohtani $^{4}$, Atsuko Nakazawa ${ }^{1}$, Mureo Kasahara ${ }^{7}$, and Akihiro Umezawa ${ }^{1 *}$

${ }^{1}$ Center for Regenerative Medicine, National Center for Child Health and Development

Research Institute, Tokyo, 157-8535, Japan

${ }^{2}$ Graduate school of Medical and Dental Sciences, Tokyo Medical and Dental University,

Tokyo, 113-0034, Japan

${ }^{3}$ Research team for Geriatric Medicine (Vascular Medicine), Tokyo Metropolitan Institute of

Gerontology, Tokyo, 173-0015, Japan

${ }^{4}$ Department of Applied Biological Science, Tokyo University of Science, Tokyo, 162-8601, Japan

${ }^{5}$ Department of Developmental and Regenerative Biology, Medical Research Institute, Tokyo

Medical and Dental University, Tokyo, 113-0034, Japan

${ }^{6}$ Department of BioSciences, Kitasato University School of Science, Kanagawa, 252-0373,

Japan

${ }^{7}$ Organ Transplantation Center, National Center for Child Health and Development, Tokyo, 157-8535, Japan

*Correspondence should be directed to:

Akihiro Umezawa,

Center for Regenerative Medicine

National Center for Child Health and Development Research Institute

2-10-1 Okura, Setagaya,

Tokyo, 157-8535, JAPAN

Phone: $\quad+81-3-5494-7047$

Fax: $\quad+81-3-5494-7048$

E-mail: $\quad$ umezawa@1985.jukuin.keio.ac.jp 


\begin{abstract}
Many drugs have the potential to induce the expression of drug-metabolizing enzymes, particularly cytochrome P450 3A4 (CYP3A4). Hepatocytes are often employed to evaluate drug-mediated CYP3A4 induction, but the variation between different cell lots is an issue that needs to be solved. Only a limited number of immortalized hepatocyte cell lines have been reported to date. In this study, we describe the successful generation of hepatocytes from disease-specific induced pluripotent stem cells (iPSCs) derived from a patient with fulminant hepatitis (FH-iPSCs). To examine the CYP3A4 induction potential, FH-iPSCs were induced into hepatocytes. Drug-mediated induction testing revealed that HepaKI exhibited a 57.2-fold increase in CYP3A4 after exposure to rifampicin, relative to control cells. These results suggest that FH-iPSCs are a preferred cell source for in vitro CYP3A4 induction assays.
\end{abstract}




\section{INTRODUCTION}

Preclinical testing of low molecular weight drugs for hepatotoxicity has traditionally been carried out in animal models such as rats and mice (Food and Drug Administration, 2012). Primary human hepatocytes can also be used for such pharmaceutical testing, but their drawbacks in drug screening include limited supply of cell lots and large variations between the lots due to genetic and environmental backgrounds. To solve the problem of lot variation, HepG2 cells have also been used to examine hepatotoxicity, because of their clonal nature. HepaRG, another hepatocyte-like clonal line established from a hepatoblastoma has the advantage of high inducibility of cytochrome p450 genes. We hypothesized that the technology to create induced pluripotent stem cells (iPSCs) would enable us to obtain hepatocytes from patients with drug-induced liver injury and establish a new and superior cell type for preclinical testing.

iPSCs have impacted numerous medical fields including clinical therapy development, drug discovery, research on inherited diseases and studies on reprogramming of differentiated cells (De Assuncao et al., 2015; Hankowski et al., 2011; Santostefano et al., 2015; Takahashi and Yamanaka, 2006). Human iPSCs also prove valuable for toxicology testing. For example, iPSC-derived hepatocytes have been shown to serve as an in vitro tool in drug metabolism and toxicology (Gripon et al., 2002; Takayama et al., 2012). iPSC-derived hepatocytes or hepatocyte-like cells can be obtained from the same origin repeatedly due to immortality of iPSCs (Holmgren et al., 2014; Lu et al., 2015; Sirenko et al., 2014). Although it is expected that hepatocytes differentiated from iPSCs can be utilized in drug toxicity testing, the actual applicability of iPSC-derived hepatocytes in this context has not yet been thoroughly examined. In this study, we generated iPSCs from a pediatric patient with hepatic failure, presumably drug-induced fulminant hepatitis, and investigated in vitro hepatotoxicity of 
various drugs. To generate mature hepatocytes that would be applicable for drug toxicity testing, we manufactured HepaKI from drug-induced hepatitis-derived iPSCs by improvement and optimization of a previously used differentiation method. HepaKI induced cyp3A4 57.2-fold upon exposure to rifampicin, suggesting that HepaKI can serve as a useful source for in vitro hepatic toxicology testing.

\section{RESULTS}

\section{Generation of drug-induced hepatic injury iPSCs}

We generated iPSCs from a patient with hepatic failure by Sendai virus infection-mediated expression of OCT4/3, SOX2, KLF4, and c-MYC (Figure 1A). When these reprogramming factors were introduced into $2.0 \times 10^{5}$ cells $(\operatorname{Hep}(\mathrm{c})$ cell) from this patient, 11 clones of iPSCs were successfully generated and designated as iPSC-K (clones \#12, \#19, \#24, \#25, \#41, \#47, \#53, \#62, \#66, \#100,\#116; Figure 1B, C). The efficiency of the iPSC colony generation was low compared to that of undamaged human cells from various adult tissues. Morphological characteristics of iPSC-K, i.e. flat and aggregated colonies, were similar to those of normal iPSCs and ESCs (Figure 1C). RT-PCR analysis revealed elimination of the Sendai virus (Figure 1D, E). Immunocytochemical analyses demonstrated expression of the pluripotent cell-specific markers, i.e. SSEA-4, TRA-1-60, SOX2, NANOG, and OCT4/3, which was consistent with the profile observed in iPSCs (Figure 1F).

To investigate multipotency in vitro, iPSCs were differentiated into ectodermal, mesodermal, and endodermal lineages. The differentiation of iPSC-K was confirmed by immunostaining using antibodies against TUJ1, $\alpha$-SMA, and AFP as ectodermal, mesodermal, and endodermal markers, respectively (Figure 1G, H). To address whether the iPSC-K have the competence to differentiate into specific tissues in vivo, teratomas were formed by 
implantation of iPSC-K in the subcutaneous tissue of immunodeficient NOD/SCID mice. iPSC-K produced teratomas within 6-10 weeks after implantation. Histological analysis of paraffin-embedded sections demonstrated that the three primary germ layers were generated as shown by the presence of ectodermal, mesodermal, and endodermal tissues in the teratoma (Figure 1I), implying iPSC-K has potential for multilineage differentiation in vitro and in vivo. Among iPSC-K clones, \#25, \#66, and \#100 generated larger area of liver-like tissues in the teratomas, while the other clones did not.

\section{Hepatic differentiation}

We investigated the efficiency of hepatic differentiation of iPSC-K by two different methods Protocol H and Protocol S (described in detail in Figure 2A, B, C). iPSC-K exhibited hepatocyte-like morphology, i.e. a polygonal and/or cuboidal shape that had tight cell-cell contact when generated by either method (Figure 2D, E) and the expression of the hepatocyte-associated genes were comparable. We thus employed the Saeko method hereafter for the iPSC-K experiments. Quantitative analysis revealed that iPSC-K expressed the genes for AFP, ALB and AAT 21 days after the start of induction (Figure 2F, G, H). Among the iPSC-K clones, iPSC-K\#25 exhibited morphology that most resembled primary hepatocytes, and high expression of hepatocyte-associated genes, and was therefore used for subsequent experiments. Time-course analysis revealed that similar expression levels of liver-associated genes were observed at 21, 28 and 35 days after the start of hepatic induction (Figure 2I, J).

\section{Verification of the manufacturing process}

To ensure the consistent production of hepatocyte-like cells from iPSC-K, we established a master cell bank and used a working cell bank (WCB) for starting material. We then 
developed a standard operating procedure for hepatic differentiation (Figure 3A). HepaKI developed into a cell type that was positive for both AFP and ALB (Figure 3B). Karyotypic analysis showed that the same chromosomes, without any aberration, were present in the WCB stock as parental fibroblastic cells from the patient (Figure 3C, D). Exome analysis revealed that iPSC-K had no significant single nucleotide alterations in a homozygous manner. To verify the procedure, we repeatedly manufactured and characterized hepatocytelike cells from the WCB. Immunocytochemistry confirmed that these hepatocyte-like cells were positive for CK7, CK8/18 (AE1/3) and Hep1, but negative for CD31 and CD34 (Figure $3 \mathrm{E})$.

\section{Induction of the genes for cyp1A2, 2B6, and $3 \mathrm{~A} 4$}

To investigate whether HepaKI exhibits CYP induction, we exposed HepaKI to omeprazole, phenobarbital, and rifampicin for 24, 48, and 48 h, respectively (Figure 4). Expression of the genes for AFP and ALB was unchanged with exposure to these drugs. Cyp1A2 was increased after exposure to omeprazole and phenobarbital, while Cyp2B6 remained unchanged (Figure 4C, D). Interestingly, Cyp3A4 was up-regulated 57.2-fold, on average, upon exposure to rifampicin (Figure 4E).

\section{DISCUSSION}

In this study, we succeeded in promoting hepatocytic differentiation from fulminant hepatitis iPSCs. We also were able to reduce the culture period to less than 30 days through EB formation, and extended the window for hepatic differentiation to more than 30 days. The reduction of the culture time and the increase in the window for differentiation could decrease manufacturing costs, which is an important requirement for efficient generation of HepaKI. Moreover, we found that HepaKI showed marked drug-mediated CYP3A4 induction, much 
higher than previously reported hepatocyte-related cells such as HepG2, HepaRG and pluripotent stem cell-derived hepatocytes.

\section{Advantages and limitations of HepaKI}

Because of its extremely high CYP3A4 induction, HepaKI is a superior cell system for toxicology testing. However, further maturation into hepatocytes is required because their hepatic characteristics, such as drug metabolism capacity, are lower than those of primary hepatocytes; the CYP2B6 and CYP3A4 genes are expressed at about one-tenth of the level seen in HepaKI. The major advantage of the manufacturing process of HepaKI is the long window of time available forcytochrome p450 induction tests. After approximately 30 days of differentiation, HepaKI cells remained stable as measured by hepatotoxicity assays, suggesting that HepaKI provides a better cost/performance balance.

\section{Modeling of drug-mediated CYP3A4 induction by HepaKI}

Because CYP3A4 contributes to the first-pass metabolism of many commercial drugs, it is important to investigate the CYP3A4-mediated hepatic metabolism in order to estimate hepatotoxicity. It is known that CYP3A4 expression in hepatocytes can be induced by various drugs, such as dexamethasone, PB, RIF, and 1 $\alpha, 25$-dihydroxyvitamin D3. The induction of CYP3A4 expression in hepatocytes by such drugs might affect the pharmacokinetics of concomitant drugs administered orally. Therefore, a HepaKI model that could evaluate drugmediated CYP3A4 induction in hepatocytes would be useful for drug discovery.

The mechanism by which the high induction of cyp3A4 (57.2-fold, on average) is achieved in HepaKI is unknown. The most likely explanation is the cell origin; iPSC-K was generated from a patient with drug-induced hepatitis, and the patient may have undergone a specific 
genetic alteration/mutation causing the high induction. Exome analysis revealed that HepaKI

had a number of single-nucleotide alterations, both homozygous and heterozygous, but we failed to find significant null alterations related to drug-induced hepatic injury or high

CYP3A4 induction. In addition, continuous generation of iPSCs from other patients with drug-induced fulminant hepatitis is necessary to produce a variety of cell sources with different genetic backgrounds for in vitro CYP3A4 induction testing and in vitro hepatotoxicity tests.

\section{MATERIALS AND METHODS}

\section{Ethical statement}

Human cells in this study were obtained in full compliance with the Ethical Guidelines for Clinical Studies (Ministry of Health, Labor, and Welfare, Japan). The cells were deposited to RIKEN Cell Bank. Animal experiments were performed according to protocols approved by the Institutional Animal Care and Use Committee of the National Research Institute for Child Health and Development.

\section{Cells}

Cells were obtained from a patient with hepatic failure $(\operatorname{Hep}(\mathrm{c}))$. The cells were maintained in Dulbecco's modified Eagle's medium (DMEM, SIGMA D6429) supplemented with 20\% FBS at $37^{\circ} \mathrm{C}$ in a humidified atmosphere containing $95 \%$ air and $5 \% \mathrm{CO}_{2}$. When the cultures reached subconfluence, the cells were harvested with Trypsin-EDTA solution (cat\# 23315, IBL CO., Ltd, Gunma, Japan), and re-plated at a density of $5 \times 10^{5}$ cells in a $100-\mathrm{mm}$ dish. Medium changes were carried out twice a week thereafter. 


\section{Generation of iPSCs}

iPSCs were generated from the patient-derived cells through reprogramming by Sendai virus infection-mediated expression of OCT4/3, SOX2, KLF4, and c-MYC as previously described (Nishimura et al., 2011). Edom22iPS\#S31 menstrual blood-derived cells were used for comparison purposes (Cui et al., 2007; Hida et al., 2008). Human iPSCs were maintained on irradiated MEFs as previously described (Makino et al., 2009; Nishino et al., 2011).

Elimination of Sendai virus was confirmed by RT-PCR. Cells just after infection served as a positive control. Sequences of the primer sets for the Sendai virus are shown in Table 1.

\section{Immunocytochemical analysis}

Cells were fixed with $4 \%$ paraformaldehyde in PBS for 10 min at $4{ }^{\circ} \mathrm{C}$. After washing with PBS and treatment with $0.2 \%$ Triton X-100 in PBS for 10 min, cells were pre-incubated with blocking buffer (10\% goat serum in PBS) for $30 \mathrm{~min}$ at room temperature, and then exposed to primary antibodies in blocking buffer overnight at $4^{\circ} \mathrm{C}$. Following washing with $0.2 \%$ PBST, cells were incubated with secondary antibodies; either anti-rabbit or anti-mouse IgG conjugated with Alexa 488 or 546 (1:300) (Invitrogen) in blocking buffer for 30 min at room temperature. Then, the cells were counterstained with DAPI and mounted.

\section{Karyotypic analysis}

Karyotypic analysis was contracted out to Nihon Gene Research Laboratories Inc. (Sendai, Japan). Metaphase spreads were prepared from cells treated with $100 \mathrm{ng} / \mathrm{mL}$ of Colcemid (Karyo Max, Gibco Co. BRL) for 6 h. The cells were fixed with methanol:glacial acetic acid (2:5) three times, and placed onto glass slides (Nihon Gene Research Laboratories Inc.). Chromosome spreads were Giemsa banded and photographed. A minimum of 10 metaphase spreads were analyzed for each sample, and karyotyped using a chromosome imaging 
analyzer system (Applied Spectral Imaging, Carlsbad, CA).

\section{Teratoma formation}

iPSCs were harvested by Accutase treatment, collected into tubes, and centrifuged. The cell pellets were suspended in the iPSellon medium. The same volume of Basement Membrane Matrix (354234, BD Biosciences) was added to the cell suspension. The cells $\left(>1 \mathrm{x} 10^{7}\right.$ ) were subcutaneously inoculated into immunodeficient, non-obese diabetic (NOD)/severe combined immunodeficiency (SCID) mice (CREA, Tokyo, Japan). The resulting tumors were dissected and fixed with PBS containing 4\% paraformaldehyde. Paraffin-embedded tissue was sliced and stained with hematoxylin and eosin. The operation protocols were approved by the Laboratory Animal Care and the Use Committee of the National Research Institute for Child and Health Development, Tokyo.

\section{Hepatic differentiation}

To generate embryoid bodies (EBs), iPSCs ( 1 x 10^4/well) were dissociated into single cells with accutase (Thermo Scientific, MA, USA) after exposure to the rock inhibitor (Y-27632: A11105-01, Wako, Japan), and cultivated in the 96-well plates (xxxxx) in the EB medium [76\% Knockout DMEM, 20\% Knockout Serum Replacement (Life Technologies, CA, USA), 2 mM GlutaMAX-I, 0.1 mM NEAA, Pen-Strep, and $50 \mu \mathrm{g} / \mathrm{mL}$ 1-ascorbic acid 2-phosphate (Sigma-Aldrich, St. Louis, MO, USA)] for 10 days. The EBs were transferred to the 24-well plates coated with collagen type $(\mathrm{xxxxx})$, and cultivated in the XF32 medium [85\% Knockout

DMEM, 15\% Knockout Serum Replacement XF CTS (XF-KSR; Life Technologies), 2 mM

GlutaMAX-I, 0.1 mM NEAA, Pen-Strep, 50 g/mL L-ascorbic acid 2-phosphate (SigmaAldrich, St. Louis, MO, USA), 10 ng/mL heregulin-1 $\beta$ (recombinant human NRG-beta 1/HRG-beta 1 EGF domain; R\&D Systems, Minneapolis, MN, USA), 200 ng/mL 
recombinant human IGF-1 (LONG R ${ }^{3}$-IGF-1; Sigma-Aldrich), and $20 \mathrm{ng} / \mathrm{mL}$ human bFGF (Life Technologies)] for 14 to 35 days.

\section{Implantation into immunodeficient mice}

HepaKI cells were harvested by treatment with Liberase, collected into tubes, centrifuged, and resuspended in PBS $(200 \mu \mathrm{l})$. The same volume $(200 \mu \mathrm{l})$ of Matrigel was added to the cell suspension. The cells $\left(>1 \times 10^{\wedge} 7\right)$ were inoculated into the subcapsular space of the kidney in severe combined immunodeficiency (SCID) mice (CREA, Tokyo, Japan). The resulting tumors were dissected and fixed with PBS containing 4\% paraformaldehyde. Paraffin-embedded tissue was sliced and stained with HE. The operation protocols were accepted by the Laboratory Animal Care and the Use Committee of the National Research Institute for Child and Health Development, Tokyo.

\section{Immunohistochemistry}

Subcutaneous nodules on mouse backs were fixed in $20 \%$ formalin and embedded in paraffin. Cut paraffin sections were deparaffinized, dehydrated, and treated with $2 \%$ proteinase $\mathrm{K}$ (Dako) in Tris-HCl buffer solution ( $\mathrm{pH} 7.5$ ) for $5 \mathrm{~min}$ at room temperature, or heated in ChemMate Target Retried Solution (Dako) for 5-20 min in a high-pressure steam sterilizer for epitope unmasking. After washing with distilled water, samples were placed in $1 \%$ hydrogen peroxide/methanol for 15 min to block endogenous peroxidase. The sections were then incubated at room temperature for $60 \mathrm{~min}$ in primary antibodies diluted with antibody diluent (Dako). The following primary antibodies against various human differentiation antigens were used: vimentin (V9, M0725, Dako, Glostrup, Denmark), albumin (Dako) and AE1/AE3 (712811, NICHIREI). Then, they were washed three times with $0.01 \mathrm{M}$ Tris buffered saline (TBS) solution (pH 7.4) and incubated with goat anti-mouse or anti-rabbit immunoglobulin labeled with dextran molecules and horseradish peroxidase (EnVision, 
Dako) at room temperature for $30 \mathrm{~min}$. After three times washes with TBS, they were incubated in 3,3'-diaminobenzidine in substrate-chromogen solution (Dako) for 5-10 min.

Negative controls were performed by omitting the primary antibody. The sections were counterstained with hematoxylin.

\section{CYP3A4 induction test}

HepaKI cells were treated with $50 \mu \mathrm{M}$ omeprazole for $24 \mathrm{~h}$ and with $500 \mu \mathrm{M}$ phenobarbital (PB, Wako) and $20 \mu \mathrm{M}$ rifampicin (RIF, Wako) for $48 \mathrm{~h}$. Controls were treated with DMSO (final concentration $0.2 \%$ ).

\section{Statistical analysis}

Statistical analysis was performed using the unpaired two-tailed Student's t test. 


\section{Funding information}

This research was supported by AMED; by KAKENHI; by the Grant of National Center for Child Health and Development. Computation time was provided by the computer cluster HA8000/RS210 at the Center for Regenerative Medicine, National Research Institute for Child Health and Development.

\section{Acknowledgements}

We would like to express our sincere thanks to N. Ito and K. Miyado for fruitful discussion, to M. Ichinose for providing expert technical assistance, to C. Ketcham for English editing and proofreading, and to E. Suzuki and K. Saito for secretarial work.

\section{Competing financial interests}

AU is a co-researcher with MTI Ltd., Terumo Corp., BONAC Corp., Kaneka Corp., CellSeed Inc., ROHTO Pharmaceutical Co., Ltd., SEKISUI MEDICAL Co., Ltd., Metcela Inc., PhoenixBio Co., Ltd., Dai Nippon Printing Co., Ltd. AU is a stockholder of TMU Science Ltd., Morikuni Ltd., and Japan Tissue Engineering Co., Ltd. The other authors declare that there is no conflict of interest regarding the work described herein. All authors have read and approved the manuscript.

\section{Author Contribution Statement}

AU designed experiments. KI, MT, SY, and TY performed experiments. KI and AU analyzed data. KI, MYI, KT, AN, and MK contributed reagents, materials and analysis tools. MT, HA, $\mathrm{HN}$, TK, and NO discussed the data and manuscript. AU and KI wrote this manuscript. 
bioRxiv preprint doi: https://doi.org/10.1101/2021.11.30.470519; this version posted November 30, 2021. The copyright holder for this preprint (which was not certified by peer review) is the author/funder, who has granted bioRxiv a license to display the preprint in perpetuity. It is made available under aCC-BY 4.0 International license.

\section{Additional Information}

The read data have been submitted to the Sequence Read Archive (SRA) under accession number SRP058607. 


\section{Reference}

Food and Drug Administration. (2012). Guidance for Industry Drug Interaction Studies:

Study Design, Data Analysis, Implications for Dosing, and Labeling Recommendations.

Cui, C.H., Uyama, T., Miyado, K., Terai, M., Kyo, S., Kiyono, T., and Umezawa, A. (2007).

Menstrual blood-derived cells confer human dystrophin expression in the murine model of

Duchenne muscular dystrophy via cell fusion and myogenic transdifferentiation. Mol Biol

Cell 18, 1586-1594.

De Assuncao, T.M., Sun, Y., Jalan-Sakrikar, N., Drinane, M.C., Huang, B.Q., Li, Y., Davila, J.I., Wang, R., O'Hara, S.P., Lomberk, G.A., et al. (2015). Development and characterization of human-induced pluripotent stem cell-derived cholangiocytes. Lab Invest 95, 684-696.

Gripon, P., Rumin, S., Urban, S., Le Seyec, J., Glaise, D., Cannie, I., Guyomard, C., Lucas, J., Trepo, C., and Guguen-Guillouzo, C. (2002). Infection of a human hepatoma cell line by hepatitis B virus. Proc Natl Acad Sci U S A 99, 15655-15660.

Hankowski, K.E., Hamazaki, T., Umezawa, A., and Terada, N. (2011). Induced pluripotent stem cells as a next-generation biomedical interface. Lab Invest 91, 972-977.

Hida, N., Nishiyama, N., Miyoshi, S., Kira, S., Segawa, K., Uyama, T., Mori, T., Miyado, K., Ikegami, Y., Cui, C., et al. (2008). Novel cardiac precursor-like cells from human menstrual blood-derived mesenchymal cells. Stem Cells 26, 1695-1704.

Holmgren, G., Sjögren, A.K., Barragan, I., Sabirsh, A., Sartipy, P., Synnergren, J., Björquist, P., Ingelman-Sundberg, M., Andersson, T.B., and Edsbagge, J. (2014). Long-term chronic toxicity testing using human pluripotent stem cell-derived hepatocytes. Drug Metab Dispos 42, 1401-1406.

Lu, J., Einhorn, S., Venkatarangan, L., Miller, M., Mann, D.A., Watkins, P.B., and LeCluyse, E. (2015). Morphological and Functional Characterization and Assessment of iPSC-Derived Hepatocytes for In Vitro Toxicity Testing. Toxicol Sci 147, 39-54.

Makino, H., Toyoda, M., Matsumoto, K., Saito, H., Nishino, K., Fukawatase, Y., Machida, 
M., Akutsu, H., Uyama, T., Miyagawa, Y., et al. (2009). Mesenchymal to embryonic incomplete transition of human cells by chimeric OCT4/3 (POU5F1) with physiological coactivator EWS. Exp Cell Res 315, 2727-2740.

Nishimura, K., Sano, M., Ohtaka, M., Furuta, B., Umemura, Y., Nakajima, Y., Ikehara, Y., Kobayashi, T., Segawa, H., Takayasu, S., et al. (2011). Development of defective and persistent Sendai virus vector: a unique gene delivery/expression system ideal for cell reprogramming. J Biol Chem 286, 4760-4771.

Nishino, K., Toyoda, M., Yamazaki-Inoue, M., Fukawatase, Y., Chikazawa, E., Sakaguchi, H., Akutsu, H., and Umezawa, A. (2011). DNA Methylation Dynamics in Human Induced Pluripotent Stem Cells over Time. PLoS Genet 7, e1002085.

Santostefano, K.E., Hamazaki, T., Biel, N.M., Jin, S., Umezawa, A., and Terada, N. (2015). A practical guide to induced pluripotent stem cell research using patient samples. Lab Invest 95, 4-13.

Sirenko, O., Hesley, J., Rusyn, I., and Cromwell, E.F. (2014). High-content assays for hepatotoxicity using induced pluripotent stem cell-derived cells. Assay Drug Dev Technol $12,43-54$.

Takahashi, K., and Yamanaka, S. (2006). Induction of pluripotent stem cells from mouse embryonic and adult fibroblast cultures by defined factors. Cell 126, 663-676.

Takayama, K., Inamura, M., Kawabata, K., Sugawara, M., Kikuchi, K., Higuchi, M., Nagamoto, Y., Watanabe, H., Tashiro, K., Sakurai, F., et al. (2012). Generation of metabolically functioning hepatocytes from human pluripotent stem cells by FOXA2 and HNF1 $\alpha$ transduction. J Hepatol 57, 628-636. 


\section{FIGURE LEGENDS}

\section{Figure 1. Generation of iPSCs from a patient with liver failure.}

A. Scheme of iPSC generation.

B. Phase contrast photomicrography of the parental cells (patient fibroblasts from the liver).

C. Generation of iPSC clones (\#12, \#19, \#24, \#25, \#41, \#47).

D. Elimination of Sendai virus vectors in iPSC-K\#24.

E. Elimination of Sendai virus vectors in the other clones.

F. Immunocytochemistry of the pluripotency-associated markers, i.e. OCT4/3, NANOG, SOX2, SSEA4 and TRA-1-60, in iPSC-K (\#12, \#19, \#24, \#25, \#62, \#66, \#100).

G. in vitro differentiation of three germ layers of iPSC-K\#24 via EB formation and adhesion culture in the EB medium. The differentiated cells were applied to the immunocytochemical analyses for markers of ectoderm (TUJ1), mesoderm ( $\alpha$ SMA), and endoderm (AFP).

H. Neural and muscular differentiation of iPSC-K\#25 via EB formation and adhesion culture in the EB medium. The differentiated cells were applied to the immunocytochemical analyses for markers of ectoderm (TUJ1) and mesoderm $(\alpha \mathrm{SMA})$.

I. Teratoma formation after subcutaneous injection of iPSC-K\#25. iPSC-K\#25 exhibited in vitro differentiation of three germ layers such as retinal pigmented epithelium (ectoderm), cartilage (mesoderm), intestinal epithelium (endoderm), and hepatocytes (endoderm).

Figure 2. Protocols for hepatic differentiation of iPSCs.

A. Scheme of two protocols, $\mathrm{H}$ and $\mathrm{S}$. The protocols differ in the periods of the 
suspension and adherent culture for hepatic differentiation.

B. Growth medium used for the suspension culture of Protocol $\mathrm{H}$ and $\mathrm{S}$.

C. Growth medium used for the adhesion culture of Protocol $\mathrm{H}$ and $\mathrm{S}$.

D. Phase-contrast photomicrograph of the hepatocyte-like cells generated from iPSC-K \#24 with Protocol S.

E. Phase-contrast photomicrograph of the hepatocyte-like cells generated from iPSC-K \#25 with Protocol S.

F. Expression of the $\alpha$-fetoprotein (AFP) gene in the iPSC-K\#25-derived hepatocytes at Day 21 with Protocol H and S. U: Undifferentiated iPSCs, H: Protocol H, S: Protocol S.

G. Expression of the albumin (ALB) gene in the iPSC-K\#25-derived hepatocytes at Day 21 with Protocol H and S. U: Undifferentiated iPSCs, H: Protocol H, S: Protocol S.

H. Expression of the $\alpha$-antitrypsin (AAT) gene in the iPSC-K\#25-derived hepatocytes at Day 21 with Protocol H and S. U: Undifferentiated iPSCs, H: Protocol H, S: Protocol S.

I. Time-course of the AFP gene expression with Protocol S in iPSC-K\#25. RNAs were isolated at 21 days (d21), 28 days (d28), and 35 days (d35) after start of the EB formation. U: undifferentiated iPSCs without EB formation.

J. Time-course of the ALB gene expression with Protocol S in iPSC-K\#25. RNAs were isolated at 21 days (d21), 28 days (d28), and 35 days (d35) after start of the EB formation.

\section{Figure 3. Systemization of drug-mediated CYP induction test.}

A. Scheme of the operation procedure for the CYP induction test with HepaKI .

B. Immunocytochemistry of HepaKI with the antibodies to ALB (red) and AFP (green). 
C. Karyotypic analysis of parental fibroblastic cells from the patient's liver.

D. Karyotypic analysis of iPSC-K\#25.

E. Immunocytochemistry of HepaKI cells at 35 and 45 days in iPGel with the antibodies to cytokeratin 7 (CK7), cytokeratins 8/18 (AE1/3), CD34, CD31, CPS1 (Hep1), and vimentin. Bar: $100 \mu \mathrm{m}$. HE: hematoxylin and eosin stain.

Figure 4. CYP induction with exposure to omeprazole (OM), phenobarbital (PB), and rifampicin (Rif).

Expression of AFP (A), ALB (B), CYP1A2 (C), CYP2B6 (D), and CYP3A4 (E) of HepaKI with exposure to DMSO (control), OM, PB, and Rif. 
bioRxiv preprint doi: https://doi.org/10.1101/2021.11.30.470519; this version posted November 30,2021 . The copyright holder for this preprint (which was not certified by peer review) is the author/funder, who has granted bioRxiv a license to display the preprint in perpetuity. It is made available under aCC-BY 4.0 International license.

\section{Table}

Table 1. Primer sets for quantitative RT-PCR

\begin{tabular}{lllc}
\hline Gene Product & Forward $\left(5^{\prime} \rightarrow 3^{\prime}\right)$ & Reverse $\left(5^{\prime} \rightarrow 3^{\prime}\right)$ & Product size \\
\hline KOS & ATGCACCGCTACGACGTGAGCGC & ACCTTGACAATCCTGATGTGG & 528 bp \\
KLF4 & ACAAGAGAAAAACATGTATGG & CGCGCTGGCAGGGCCGCTGCTCGAC & $529 \mathrm{bp}$ \\
C-MYC & TAACTGACTAGCAGGCTTGTCG & TCCACATACAGTCCTGGATGATGATG & 532 bp \\
SeV & GGATCACTAGGTGATATCGAGC & ACCAGACAAGAGTTTAAGAGATATGTAT & $181 \mathrm{bp}$ \\
& & C & \\
\hline
\end{tabular}


Day $0 \quad$ Day $1 \quad$ Day $2 \quad$ Day 5-

\begin{tabular}{|l|l|l|l|}
\hline Preparation & Infection & Culture & Feeder cells \\
\hline
\end{tabular}

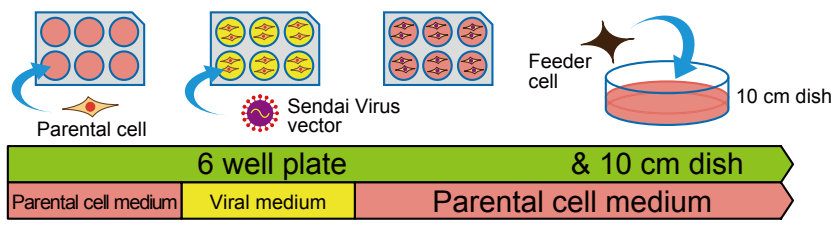

Day 6- Day 7- Day 13- Day 15-

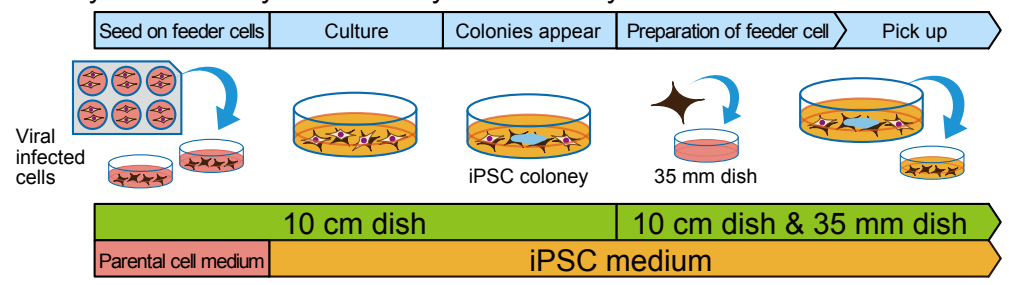

D

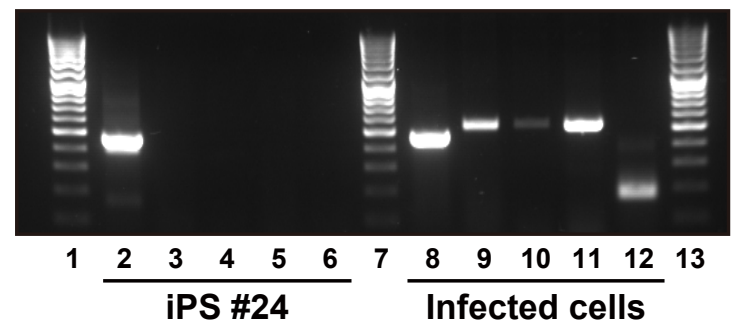

E

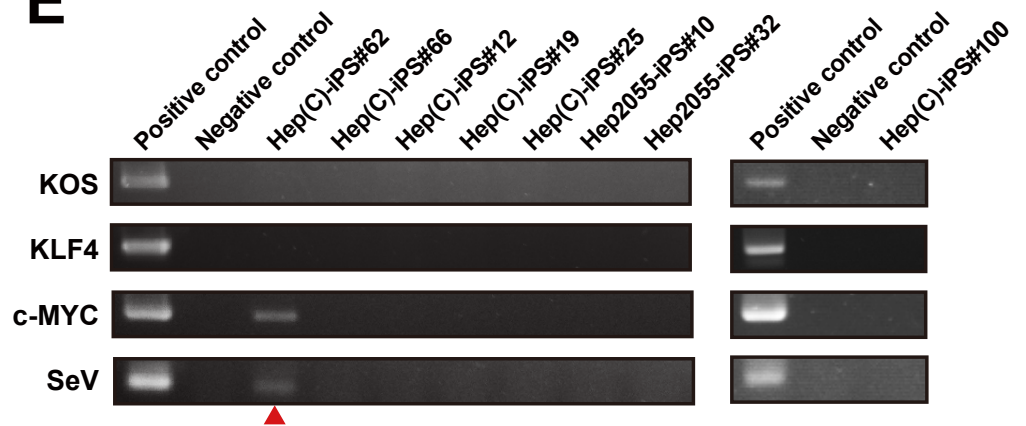

G
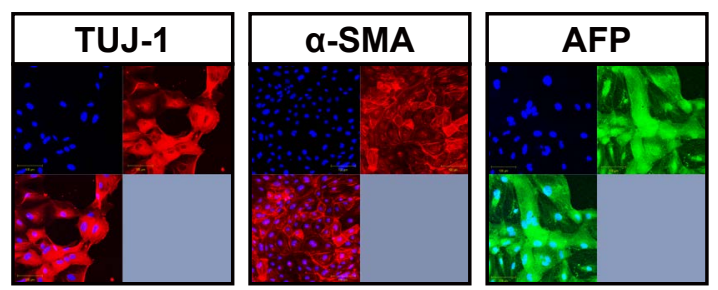

H
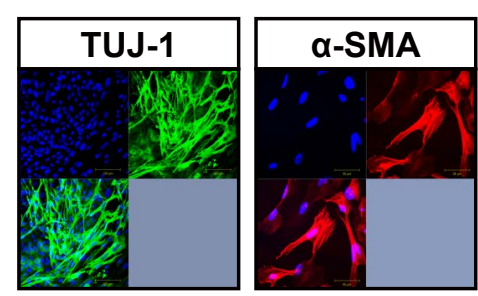

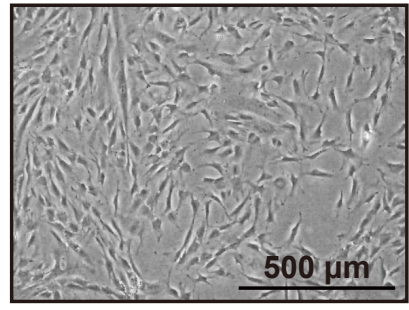

C
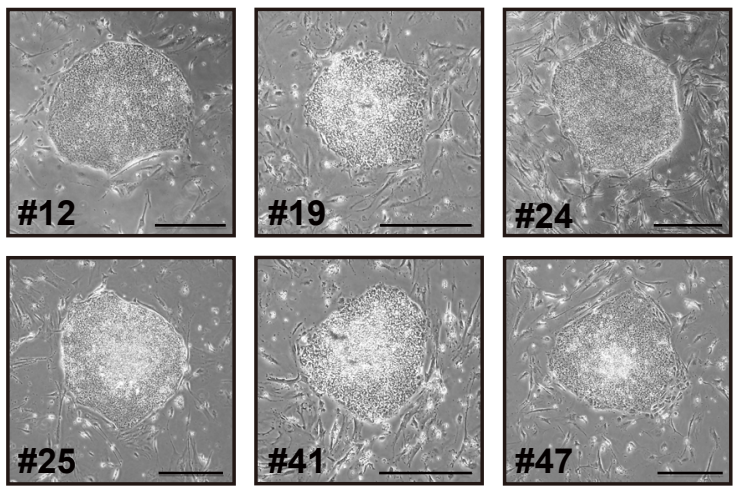

F
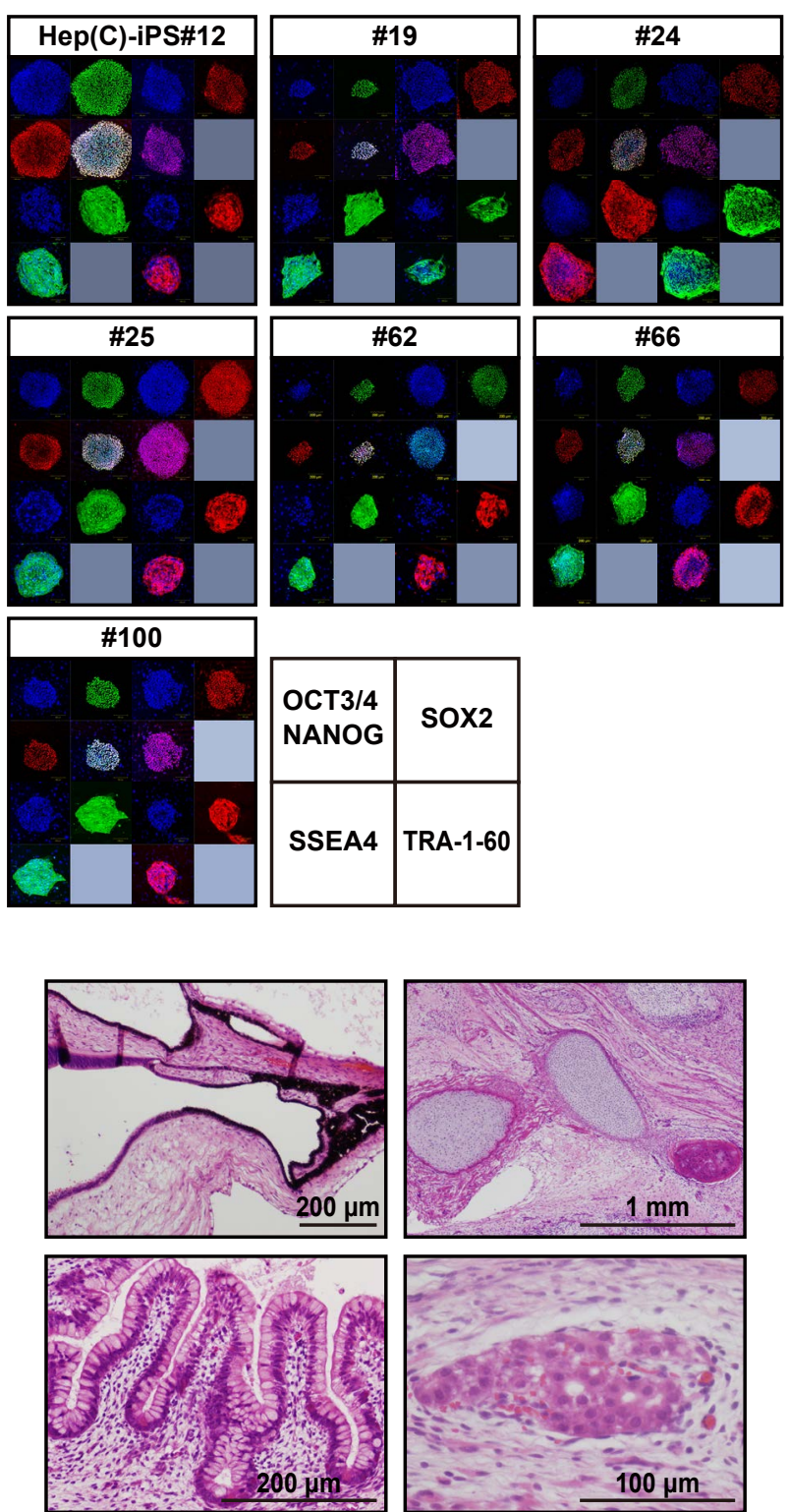

Figure 1 
bioRxiv preprint doi: https://doi.org/10.1101/2021.11.30.470519; this version posted November 30, 2021. The copyright holder for this preprint (which was not certified by peer review) is the author/funder, who has granted bioRxiv a license to display the preprint in perpetuity. It is made

A

Protocol H

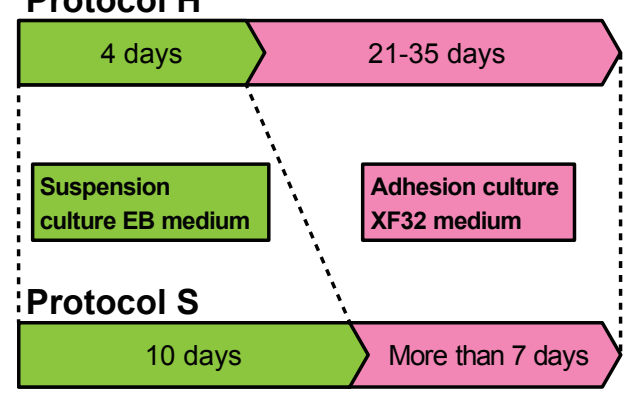

available under aCC-BY 4.0 International license.

C
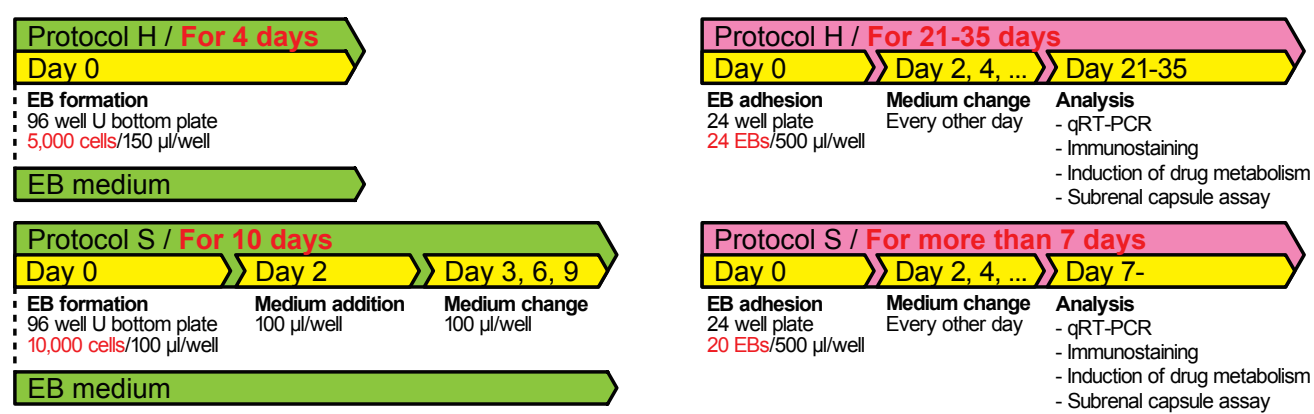

$\mathbf{F}$
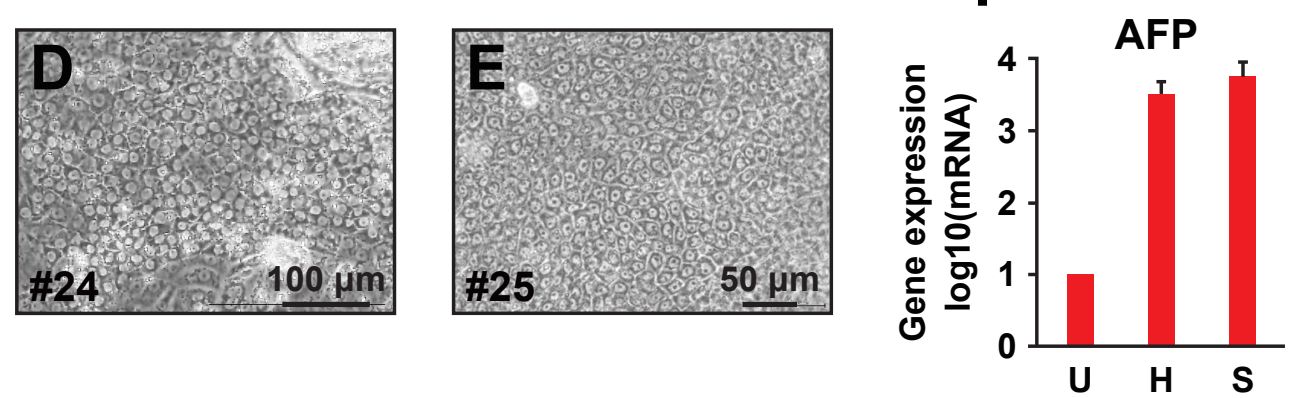

G

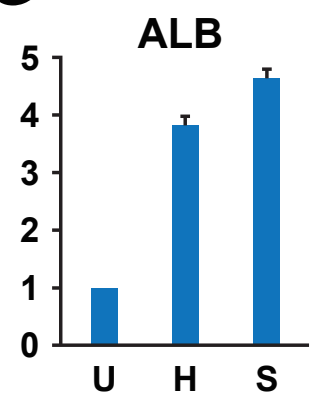

H

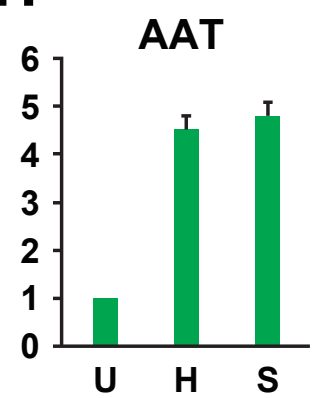

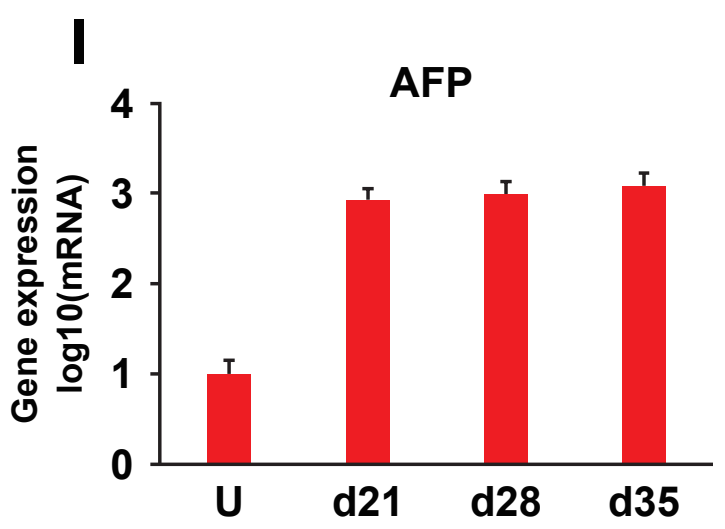

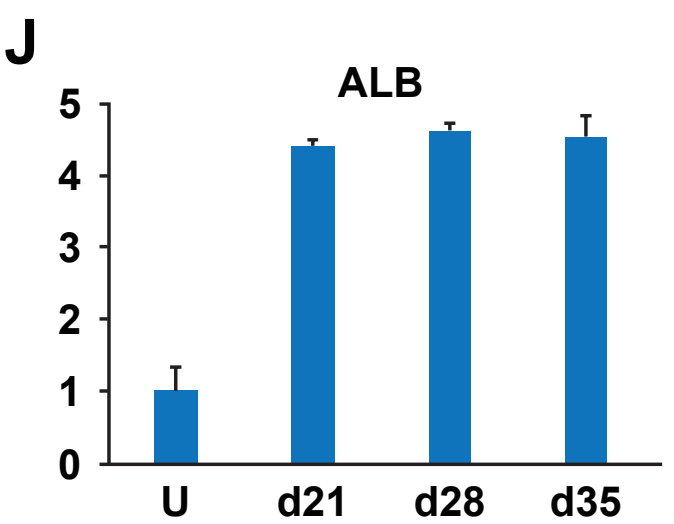

Figure 2 


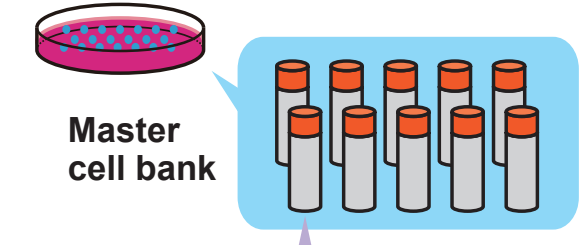

Working cell bank

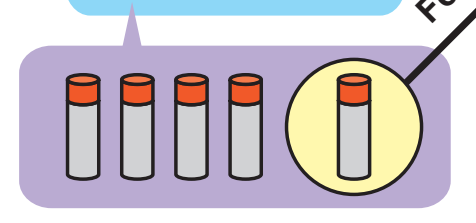

B

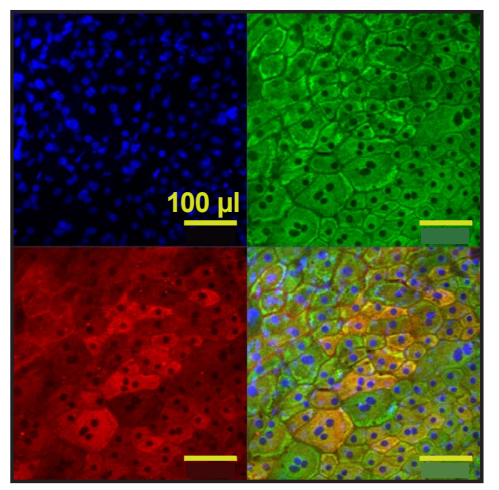

C

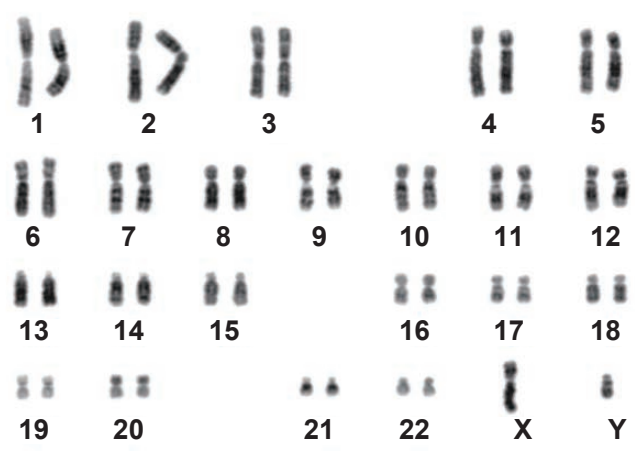

D

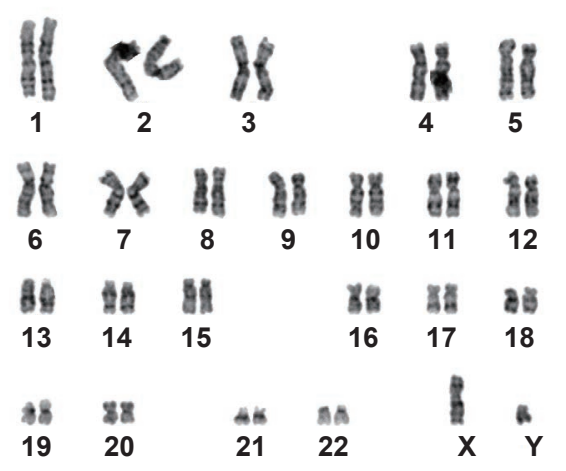

\section{E}

Suspention culture / For 10 days \begin{tabular}{|l|l|}
\hline Day 0 & Day 2 \\
Dadd medium & Day 6, 9 \\
\hline Medium change
\end{tabular} 96 well U bottom plate $100 \mu /$ well $\quad 100 \mu /$ well 10,000 cells/100 $\mu /$ well EB medium

\begin{tabular}{|lll|}
\hline \multicolumn{3}{|l|}{ Adhesion culture / For more than 7 days } \\
\hline Day 0 & Day 2 & Day $3,6,9$ \\
\hline EB adhesion & Medium change & Analysis \\
24 well plate & Every other day & - qRT PCR \\
$20 \mathrm{EBs} / 500 \mathrm{\mu l} /$ well & & - Immunostaining \\
$35 \mathrm{~mm}$ dish & - Induction of drug \\
$100 \mathrm{EBs} / 1 \mathrm{ml} / \mathrm{dish}$ & metabolism \\
(Collagen coated) & \\
\hline XF32 medium & \\
\hline
\end{tabular}
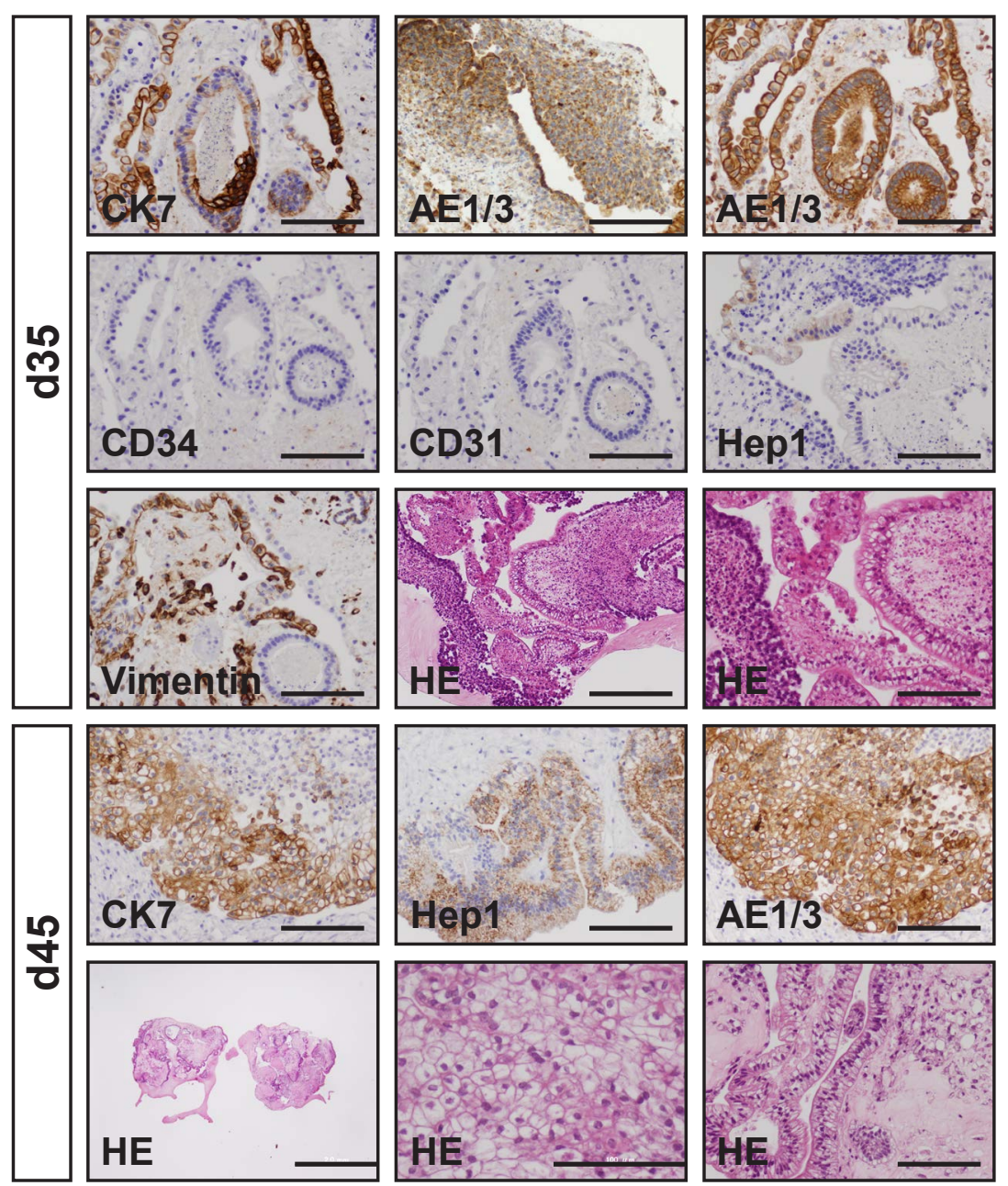
A
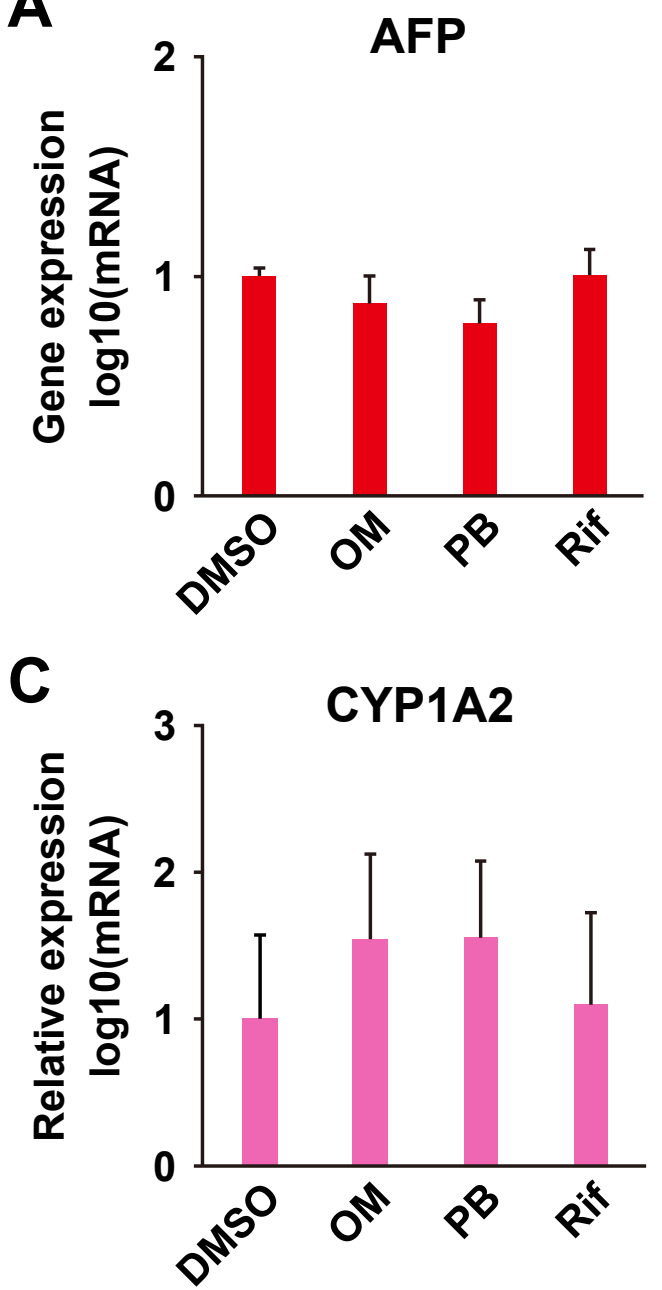

B

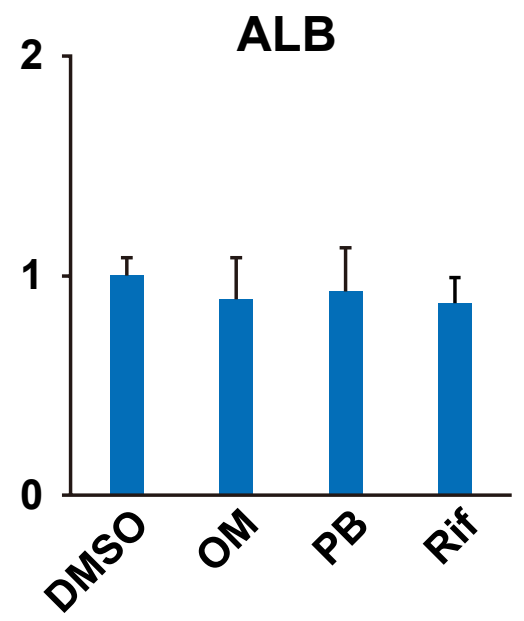

D

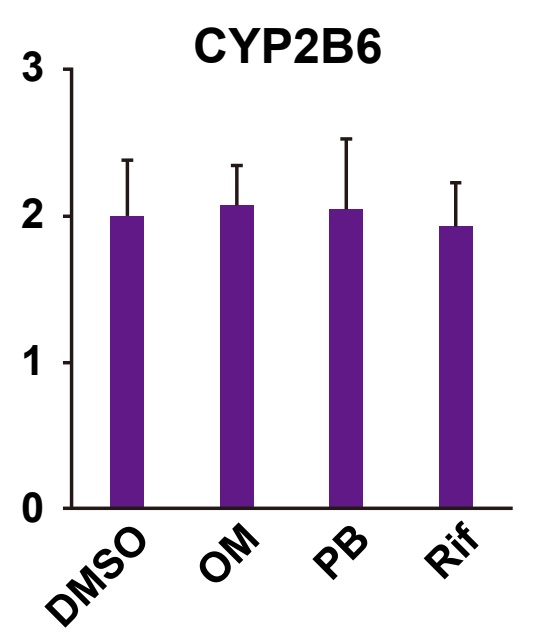

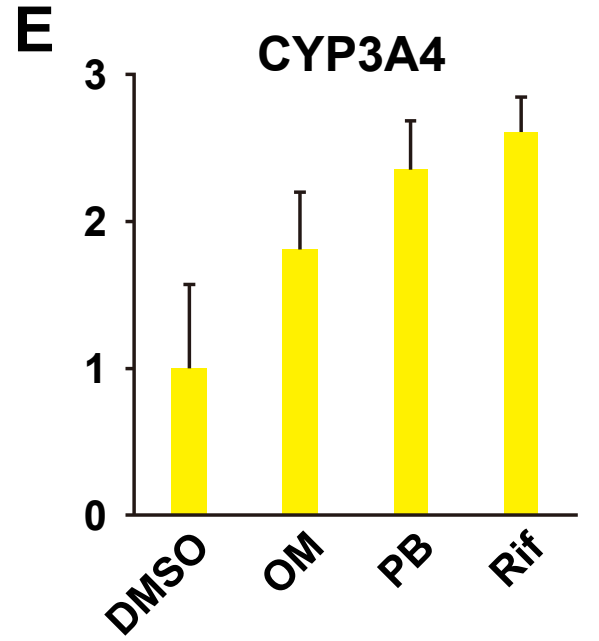

Figure 4 